\title{
Curcumin targets vascular endothelial growth factor via activating the PI3K/Akt signaling pathway and improves brain hypoxic-ischemic injury in neonatal rats
}

\author{
Jia Li ${ }^{1, \#}$, Yan $\mathrm{An}^{3, \#}$, Jia-Ning Wang ${ }^{2, *}$, Xiao-Ping Yin ${ }^{2}$, Huan Zhou ${ }^{2}$, and Yong-Sheng Wang ${ }^{4}$ \\ ${ }^{1}$ Department of Otolaryngology Head and Neck Surgery, The Second Hospital of Baoding, Baoding 071000, ${ }^{2}$ Department of Radiology, Affiliated Hospital of \\ Hebei University, Baoding 071000, ${ }^{3}$ Department of Obstetrics, Affiliated Hospital of Hebei University, Baoding 071000, ${ }^{4}$ Department of MR Room, Qingyuan \\ District People"s Hospital, Baoding 071000, China
}

\author{
ARTICLE INFO \\ Received March 17, 2020 \\ Revised May 22, 2020 \\ Accepted May 22, 2020 \\ *Correspondence \\ Jia-Ning Wang \\ E-mail:wangjianing_med@163.com
}

\section{Key Words}

Brain

Curcumin

Hypoxic-ischemic

PI3K/Akt signaling pathway

Vascular endothelial growth factor

\#These authors contributed equally to this work.

\begin{abstract}
This study aimed to evaluate the effect of curcumin on brain hypoxicischemic (HI) damage in neonatal rats and whether the phosphoinositide 3-kinase (PI3K)/Akt/vascular endothelial growth factor (VEGF) signaling pathway is involved. Brain $\mathrm{HI}$ damage models were established in neonatal rats, which received the following treatments: curcumin by intraperitoneal injection before injury, insulin-like growth factor 1 (IGF-1) by subcutaneous injection after injury, and VEGF by intracerebroventricular injection after injury. This was followed by neurological evaluation, hemodynamic measurements, histopathological assessment, TUNEL assay, flow cytometry, and western blotting to assess the expression of p-PI3K, PI3K, p-Akt, Akt, and VEGF. Compared with rats that underwent sham operation, rats with brain $\mathrm{HI}$ damage showed remarkably increased neurological deficits, reduced right blood flow volume, elevated blood viscosity and haematocrit, and aggravated cell damage and apoptosis; these injuries were significantly improved by curcumin pretreatment. Meanwhile, brain $\mathrm{HI}$ damage induced the overexpression of $\mathrm{p}-\mathrm{PI} 3 \mathrm{~K}, \mathrm{p}-\mathrm{Akt}$, and VEGF, while curcumin pretreatment inhibited the expression of these proteins. In addition, IGF-1 treatment rescued the curcumin-induced down-regulated expression of $p$ PI3K, p-Akt, and VEGF, and VEGF overexpression counteracted the inhibitory effect of curcumin on brain $\mathrm{HI}$ damage. Overall, pretreatment with curcumin protected against brain $\mathrm{HI}$ damage by targeting VEGF via the PI3K/Akt signaling pathway in neonatal rats.
\end{abstract}

\section{INTRODUCTION}

Neonatal hypoxic-ischemic encephalopathy (HIE) is a common brain injury disease in near-term infants, with high rates of morbidity, disability, and mortality [1]. Intrauterine hypoxia and intrapartum asphyxia are considered the major underlying causes of HIE and lead to tremendous detrimental effects on brain development in newborns, thereby resulting in devastating disabilities such as mental retardation, cerebral palsy, learning disabilities, and epilepsy [2-4]. Despite the increased understanding of neonatal disease pathogenesis and the intense advancements in modern treatments, the significant disability and mortality caused by HIE remain serious issues $[5,6]$. Therefore, elucidating the underlying mechanisms of neonatal HIE to search for effective therapeutic targets is essential.

Various diseases after HIE are mainly caused by long-lasting neurological deficits, and neuroprotective agents are considered potentially therapeutic for neonatal HIE [7]. It has been widely

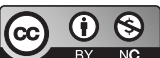

This is an Open Access article distributed under the terms of the Creative Commons Attribution Non-Commercial License, which permits unrestricted non-commercial use, distribution, and reproduction in any medium, provided the original work is properly cited. Copyright @ Korean J Physiol Pharmacol, pISSN 1226-4512, elSSN 2093-3827
Author contributions: J.L. and Y.A. wrote the manuscript. J.N.W. supervised and coordinated the study. X.P.Y., H.Z., and Y.S.W. performed the experiments. 
recognized that improving blood supply to brain tissue is closely related to the recovery of neuronal function [8]. Vascular endothelial growth factor (VEGF) is a cytokine secreted by vascular endothelial cells and is currently the most important growth factor for inducing angiogenesis [9]. It exhibits neuroprotective and neurotrophic functions via promoting proliferation and angiogenesis of vascular endothelial cells, vasodilation, and the synthesis of various vasoactive substances, as well as inhibiting vascular smooth muscle cell proliferation [9]. The application of exogenous VEGF contributes to neuronal survival in mesencephalic explant cultures [10], and VEGF is an attractive target for brain hypoxicischemic (HI) damage [11]. Thus, drugs targeting VEGF may be an effective neuroprotective agent for brain $\mathrm{HI}$ damage.

In recent years, several traditional Chinese medicines have been demonstrated to exert neuroprotective effects $[12,13]$. Curcumin, a low-molecular-weight bioactive compound isolated from turmeric (Curcuma longa), can affect various diseases by regulating oxidative stress, cell apoptosis, and the inflammatory response [14]. A recent study has suggested that curcumin can attenuate brain $\mathrm{HI}$ damage in neonatal rats [15]. However, whether curcumin improves brain HI damage via VEGF regulation is still unclear.

The phosphoinositide 3-kinase (PI3K)/Akt signal transduction pathway may be an important signaling pathway for directly regulating VEGF expression in brain microvascular endothelial cells [16]. This study aimed to evaluate the effect of curcumin on brain $\mathrm{HI}$ damage in neonatal rats and whether the effect of curcumin is associated with the PI3K/Akt/VEGF signaling pathway.

\section{METHODS}

\section{Animal model}

This study was approved by the Animal Ethics Committee of Affiliated Hospital of Hebei University prior to experimentation (no. 06ChiC 6 244016475). Healthy neonatal Sprague-Dawley rats (18-25 g, 10 days old, male) were used for the following experiments after one week of acclimation (17-days old). Brain HI damage rat models were established as previously described [17]. Briefly, the rats were placed in the induction chamber and adjust the ether to $4 \%$ to induction anesthesia and adjust the ether to $3 \%$ to maintenance, then followed by fixation on the operating table. A $0.4 \mathrm{~cm}$ cervical incision was cut to expose the arteries. Then the right common carotid artery was ligated with a 7-0 suture, and the skin incision was sutured. After $1 \mathrm{~h}$, rats were placed in an anoxic chamber containing $8 \%$ oxygen and $92 \%$ ammonia gas for $2.5 \mathrm{~h}$ (flow rate $2 \mathrm{~L} / \mathrm{min}$ ), and then returned to the normal oxygen cage. The sham operation consisted of the entire procedure but without ligation and anoxic treatment. Constant temperature was maintained throughout the operation.

\section{Animal grouping, drug administration, and sample collection}

In total, 60 neonatal rats were randomly and equally assigned to five groups: 1) the sham group: rats underwent a sham operation and received intraperitoneal injection of saline; 2) the HI group: rats with brain $\mathrm{HI}$ damage received intraperitoneal injection of saline; 3) the HI + CUR group: rats received intraperitoneal injection of $200 \mathrm{mg} / \mathrm{kg}$ curcumin $30 \mathrm{~min}$ before brain $\mathrm{HI}$ damage surgery; 4) the HI + CUR + insulin-like growth factor-1 (IGF-1) group: rats received intraperitoneal injection [18] of 200 $\mathrm{mg} / \mathrm{kg}$ curcumin $30 \mathrm{~min}$ before brain HI damage surgery and subcutaneous injection of $3 \mathrm{mg} / \mathrm{kg}$ IGF-1 $24 \mathrm{~h}$ after HI injury (Peprotech, Inc., Rocky Hill, NJ, USA) [19]; 5) the HI + CUR + ovVEGF group: rats received intraperitoneal injection of $200 \mathrm{mg} /$ $\mathrm{kg}$ curcumin 30 min before brain HI damage surgery and intracerebroventricular injection of 20 ng VEGF (Sigma, St. Louis, MO, USA) 5 min after reoxygenation [20]. Hemodynamic measurements were performed $12 \mathrm{~h}$ after treatment and the blood pressure during and after HI was monitored to exclude brain injury caused by systemic hypotension. One day ( $24 \mathrm{~h})$ after treatment, a neurological evaluation was performed and then the rats were sacrificed to obtain brain tissues.

\section{Neurological evaluation}

Neurological deficits were evaluated by Longa's method [21] and graded by different scores. Neurological function was graded according to the following scores: 0 (normal, no neurological deficits), 1 (failure to extend left forepaw fully), 2 (turning to the left while walking), 3 (dumping to the left while walking), and 4 (unconsciousness and failing to walk spontaneously).

\section{Hemodynamic measurements}

Hemodynamic parameters, including left and right cerebral hemisphere blood flow volume, blood viscosity, and hematocrit, were measured using an electromagnetic blood flowmeter (MFV3200; Nihon Kohden, Tokyo, Japan) and an auto cleaning rotating viscometer (LBY-N6A; Puli Co., Beijing, China).

\section{Histopathological assessment}

Brain tissues of cortex and hippocampus were fixed at $4^{\circ} \mathrm{C}$ for $24 \mathrm{~h}$ with $4 \%$ paraformaldehyde, embedded in paraffin, and sliced into sections. After dehydration with gradient ethanol, the sections underwent hematoxylin-eosin (H\&E) staining: hematoxylin staining, differentiation in $1 \%$ hydrochloric acid alcohol, eosin staining, and mounting with neutral resin. The sections were observed by light microscopy (Olympus, Tokyo, Japan). 


\section{2,3,5-Triphenyltetrazolium Chloride (TTC) Staining}

The brains were sectioned into spaced coronal blocks $(2 \mathrm{~mm})$. These sections were stained with 2\% TTC (Coolaber, Beijing, China) at $37^{\circ} \mathrm{C}$ for $15 \mathrm{~min}$. Images of the staining were acquired using a digital camera (EOS 90D; Canon, Tokyo, Japan). The non-infarcted area was stained red, the infarcted area was grayish white, and the area between the red area and the gray area was ischemic.

\section{TUNEL assay}

The HI brain tissue sections of the right brain were obtained as described for HE staining. Then cell apoptosis in brain tissues was evaluated by TUNEL staining using a commercial kit (Roche, Mannheim, Germany) following the manufacturer's guidelines. The sections were observed with a fluorescence microscope (Olympus).

\section{Flow cytometry}

Single-cell suspensions of brain tissues in ice-cold flow cytometry buffer (2\% fetal calf serum and $2 \%$ mouse serum in phosphate buffer saline [PBS]) were prepared. To determine the percentage of early and late apoptotic cells, single cells were stained using the following antibody set: Annexin V-FITC and PI Cells according to the manufacturer's instructions (Cat No: 556547; BD Biosciences, San Diego, CA, USA). Data were acquired on an LSRFortessa cell analyzer (BD Biosciences) and analyzed using FlowJo software (Tree Star Inc., Ashland, OR, USA).

\section{Western blotting}

The HI brain tissue of the right brain in various groups was lysed by RIPA lysis buffer (Gibco, Grand Island, NY, USA) supplemented with phenylmethanesulfonyl fluoride (1 mM, Sigma). Protein was extracted by centrifugation and detected by the BCA kit (Shanghai Sangon Biotech Co., Ltd, Shanghai, China). Then protein samples were separated on SDS-PAGE gel, transferred to polyvinylidene fluoride membranes, and blocked with $5 \%$ nonfat milk for $1 \mathrm{~h}$. Next, the membrane was incubated with anti-rat pPI3K, PI3K, p-Akt, Akt, VEGF, or glyceraldehyde-3-phosphate dehydrogenase (GAPDH) antibody (1:500, Cat: sc-32233; Santa Cruz Biotechnology, Santa Cruz, CA, USA) overnight at $4^{\circ} \mathrm{C}$, washed with PBS, and incubated with secondary antibody (1:5000, Cat:111-035-003; Jackson ImmunoResearch, West Grove, PA, USA) for $2 \mathrm{~h}$ at room temperature. Enhanced chemiluminescence (ECL, Millipore, USA) was used to detect the protein levels.

\section{Statistical analysis}

GraphPad Prism 6 software (GraphPad Software Inc., San
Diego, CA, USA) was used for statistical analysis. Data were expressed as mean \pm standard deviation and analyzed by one-way ANOVA followed by multiple comparison with the Tukey test. A value of $p<0.05$ was considered significant and $p<0.01$ was considered highly significant.

\section{RESULTS}

\section{The effect of curcumin on neurological deficits, hemodynamic changes, and histopathological changes in neonatal rats with brain HI damage}

All rats with brain HI damage exhibited depressive symptoms, mainly manifested as tonic convulsions and tremors. To investigate whether the pretreatment with curcumin could alleviate the symptoms and pathological changes of brain HI damage, neurological deficits, hemodynamic changes, and histopathological changes in neonatal rats with brain HI damage were measured. TTC staining showed that compared with HI group, the brain HI damage in HI + CUR group is improved (Fig. 1A). Longa's score results found that compared with the sham group, neurological deficit scores were significantly increased in the HI group $(\mathrm{p}<$ 0.01 , Fig. $1 \mathrm{~B}$ ), while pretreatment with curcumin significantly reduced neurological deficit scores in comparison with the HI group at $24 \mathrm{~h}$ and $72 \mathrm{~h}$ after treatment ( $<<0.01$, Fig. 1B). The hemodynamic results revealed that compared with the sham group, right blood flow volume was significantly reduced and blood viscosity and hematocrit were significantly increased in the HI group ( $p<0.05$, Table 1 ), while pretreatment with curcumin decreased blood viscosity and hematocrit $(p<0.05$, Table 1$)$. In addition, HE staining revealed that the cortical and hippocampal cells exhibited large nuclei and little cytoplasm in the sham group, whereas the cortex and hippocampus in the HI and CUR groups both exhibited different degrees of cell swelling, balloon-like changes, sparse cells, widened gaps, disordered cell arrangement, unclear structure, and nuclear pyknosis (Fig. 1C). Compared with the CUR group, cell damage was aggravated in the HI group (Fig. 1C). TUNEL staining and flow cytometry also revealed conspicuously increased apoptotic cells in the HI group compared with the sham group, while pretreatment with curcumin reduced the number of apoptotic cells ( $<<0.01$, Fig. 1D, E). Both the proportions of early and late apoptotic cells in the HI group were significantly higher than those in the sham group; however, pretreatment of curcumin significantly reduced the proportion in the HI + CUR group $(\mathrm{p}<0.01$, Fig. 1E).

\section{The effect of curcumin on the PI3K/Akt signaling pathway and VEGF in neonatal rats with brain $\mathrm{HI}$ damage}

Considering the effect of curcumin on brain HI damage, the 

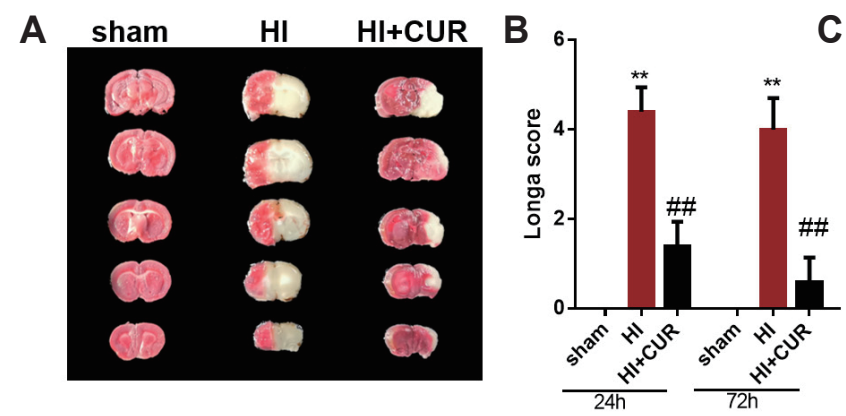

C
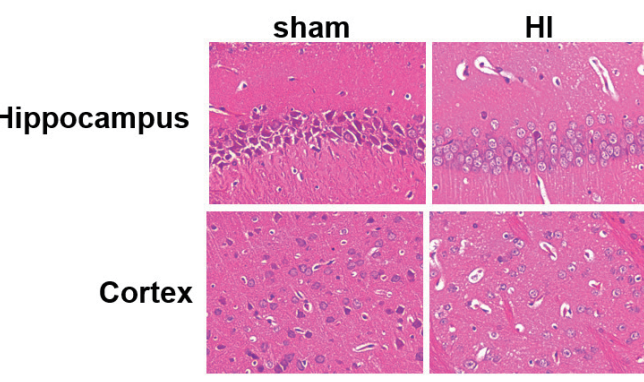

HI+CUR
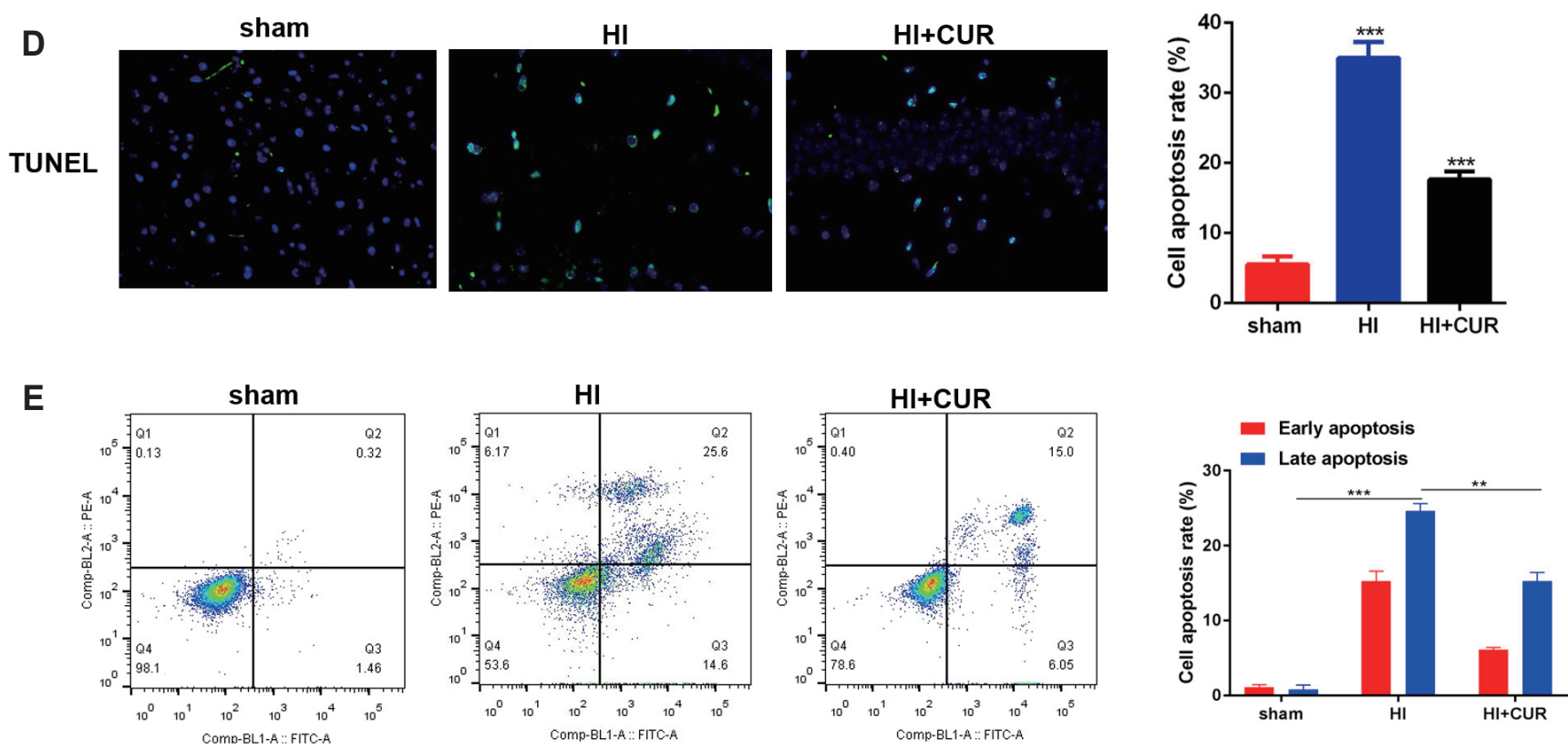

Fig. 1. Curcumin improves brain HI damage in neonatal rats ( $n=12$ per group). (A) HI damage in brain tissues of the sham, $H I$, and $H I+C U R$ groups by TUNEL staining. (B) Neurological deficits evaluated in the sham, $\mathrm{HI}$, and $\mathrm{HI}+\mathrm{CUR}$ groups by Longa's score at $24 \mathrm{~h}$ and $72 \mathrm{~h}$ after treatment. (C) Histopathological changes of brain tissues in the sham, $\mathrm{HI}$, and $\mathrm{HI}+\mathrm{CUR}$ groups by H\&E staining ( $\times 400)$. (D) Cell apoptosis in brain tissues of the sham, $\mathrm{HI}$, and $\mathrm{HI}+\mathrm{CUR}$ groups by TUNEL staining ( $\times 400)$. (E) Early and late apoptotic in brain tissues of the sham, HI, and HI + CUR groups by flow cytometry. $\mathrm{HI}$, hypoxic-ischemic injury; CUR, curcumin. ${ }^{* *} \mathrm{p}<0.01,{ }^{* * *} \mathrm{p}<0.001 \mathrm{vs}$. sham group; ${ }^{\# \#} \mathrm{p}<0.01 \mathrm{vs}$. HI group.

Table 1. Haemodynamic changes of rats in the sham, $\mathrm{HI}$, and $\mathrm{HI}+\mathrm{CUR}$ groups

\begin{tabular}{lccc}
\hline \multicolumn{1}{c}{ Parameters } & Sham group & HI group & HI + CUR group \\
\hline Left blood flow volume $(\mathrm{ml} / \mathrm{min})$ & $46.5 \pm 6.3$ & $45.8 \pm 5.3$ & $46.2 \pm 5.9$ \\
Right blood flow volume $(\mathrm{ml} / \mathrm{min})$ & $47.2 \pm 5.6$ & $43.8 \pm 4.3^{*}$ & $45.7 \pm 5.1$ \\
Blood viscosity (mPa·s) & $6.05 \pm 0.34$ & $7.62 \pm 0.29^{*}$ & $6.18 \pm 0.24^{*}$ \\
Hematocrit $(\mathrm{L} / \mathrm{L})$ & $0.385 \pm 0.002$ & $0.413 \pm 0.001^{*}$ & $0.392 \pm 0.003^{*}$ \\
\hline
\end{tabular}

$\mathrm{HI}$, hypoxic-ischemic injury; CUR, curcumin. ${ }^{*} \mathrm{p}<0.05$ vs. sham group; ${ }^{\sharp} \mathrm{p}<0.05$ vs. HI group.

expression levels of p-PI3K, p-Akt, and VEGF were analyzed to explore the mechanism of the effect of curcumin. Western blotting revealed that compared with the sham group, the protein expression of p-PI3K, p-Akt, and VEGF were significantly increased in the HI group ( $\mathrm{p}<0.01$, Fig. 2). Pretreatment with curcumin significantly reduced the expression of p-PI3K, p-Akt, and VEGF in comparison with the HI group ( $p<0.01$, Fig. 2 ).
The effect of activating the PI3K/Akt signaling pathway in curcumin-treated neonatal rats with brain HI damage

To investigate whether the PI3K/Akt signaling pathway influenced the role of curcumin, curcumin-treated HI rats were administered IGF-1. Compared with the HI + CUR group, IGF1 treatment significantly promoted p-PI3K, p-Akt, and VEGF expression ( $\mathrm{p}<0.01$, Fig. 3). 

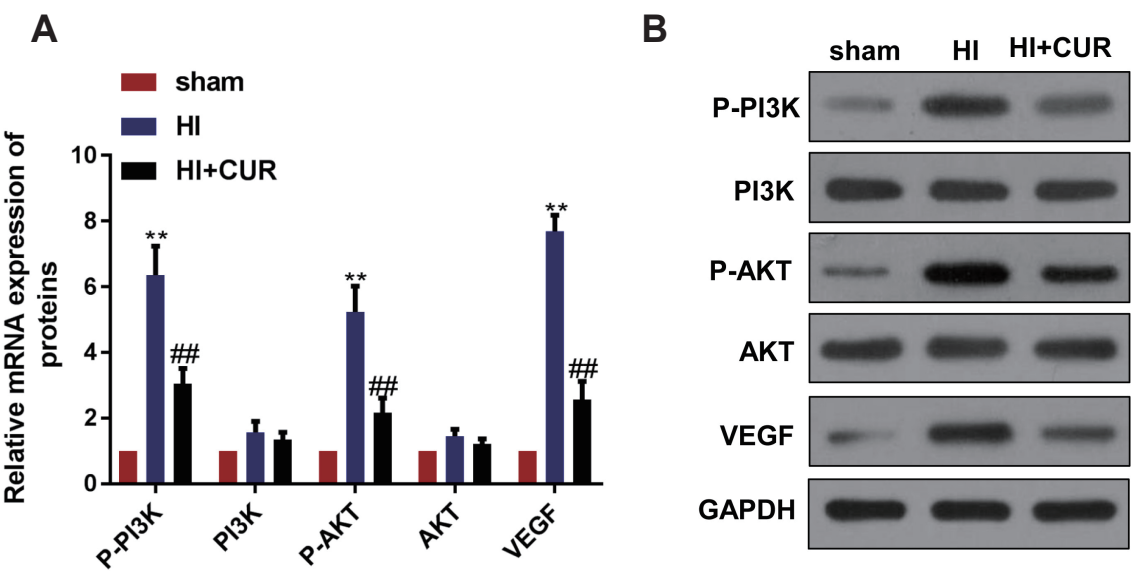

Fig. 2. Curcumin promotes the activation of PI3K/Akt signaling pathway and VEGF expression in neonatal rats with brain $\mathrm{HI}$ damage $(\mathrm{n}=12$ per group). (A) Band intensities from western blots were calculated. (B) The protein expression of p-PI3K, PI3K, p-Akt, Akt, and VEGF in the sham, HI, and $\mathrm{HI}+$ CUR groups by western blotting. HI, hypoxic-ischemic injury; CUR, curcumin; VEGF, vascular endothelial growth factor; GAPDH, glyceraldehyde-3-phosphate dehydrogenase. ${ }^{* *} \mathrm{p}<0.01$ vs. sham group; ${ }^{* \#} \mathrm{p}<0.01$ vs. HI group.

A

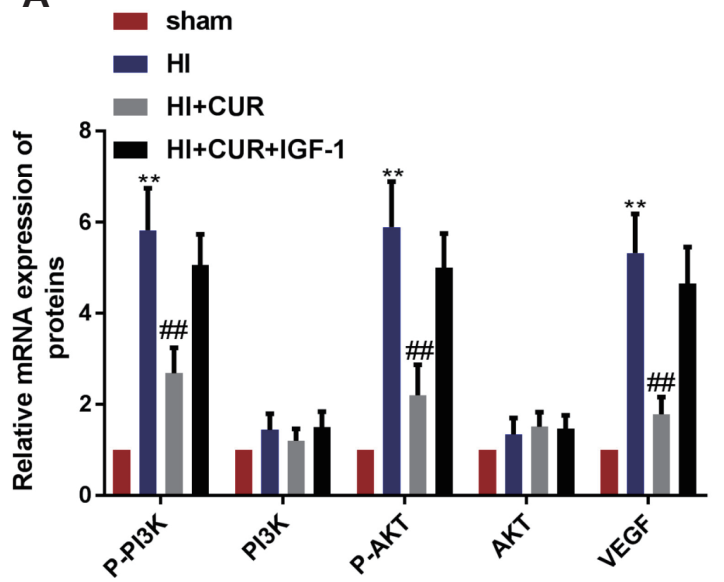

B

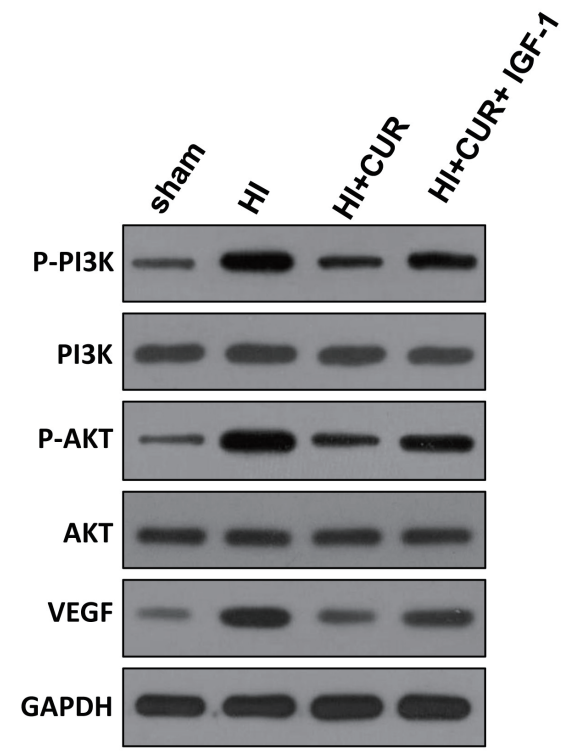

Fig. 3. Inhibitory effect of curcumin on VEGF expression disappears via activating PI3K/Akt signaling pathway in neonatal rats with brain HI damage ( $\mathbf{n}=12$ per group). (A) Band intensities from western blots were calculated. (B) The protein expression of p-PI3K, PI3K, p-Akt, Akt, and VEGF in the sham, HI, HI + CUR and HI + CUR + IGF-1 groups by western blotting. HI, hypoxic-ischemic injury; CUR, curcumin; IGF-1, insulin-like growth factor 1; VEGF, vascular endothelial growth factor; GAPDH, glyceraldehyde-3-phosphate dehydrogenase. ${ }^{* *} p<0.01$ vs. sham group; ${ }^{\# \# ~}<0.01$ vs. HI group.

\section{The effect of VEGF overexpression in curcumin- treated neonatal rats with brain $\mathrm{HI}$ damage}

To further explore the role of VEGF in curcumin-treated neonatal rats with brain $\mathrm{HI}$ damage, curcumin-treated $\mathrm{HI}$ rats were administered VEGF. Also, VEGF expression was higher in the HI + CUR + ov-VEGF group than that in the HI+CUR group (Fig. $4 \mathrm{~A})$. The results also showed that compared with the HI + CUR group, VEGF overexpression significantly increased brain HI damage (Fig. 4A), neurological deficit scores at $24 \mathrm{~h}$ and $72 \mathrm{~h}$ after treatment $(\mathrm{p}<0.01$, Fig. $4 \mathrm{C})$ and also aggravated cell damage (Fig.
4D) and cell apoptosis (Fig. 4E, F).

\section{DISCUSSION}

In the present study, a neonatal rat model of brain HI damage was successfully constructed. The results revealed that pretreatment with curcumin significantly improved neurological deficits and hemodynamic changes, attenuated histopathological injury and cell apoptosis, and decreased the number of both early and late apoptotic cells in neonatal rats with brain $\mathrm{HI}$ damage. 
A
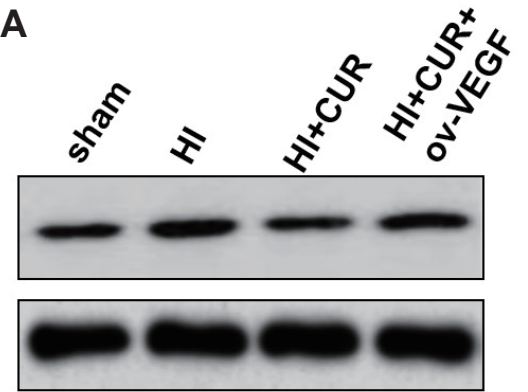

B sham HI Hl+CUR HI+CUR+ OV-VEGF

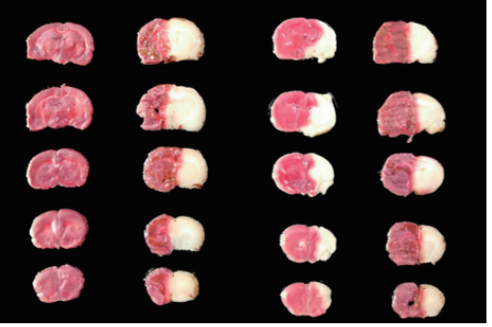

C

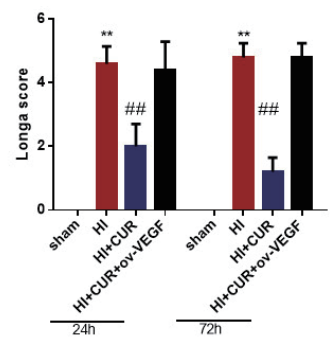

D

sham

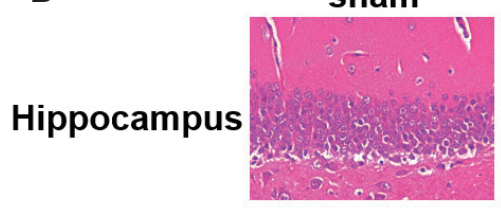

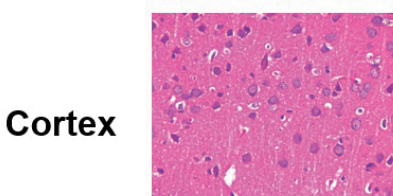

E

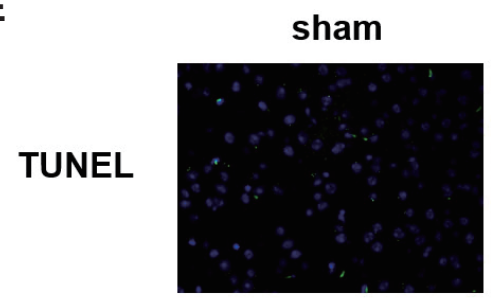

$\mathbf{F}$

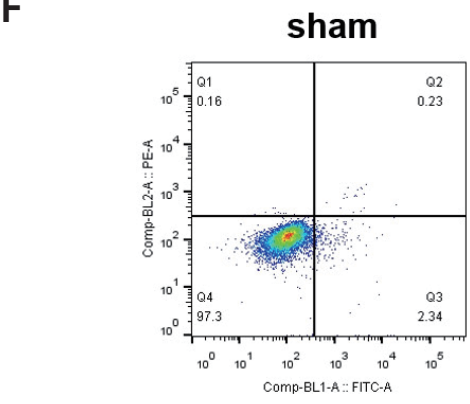

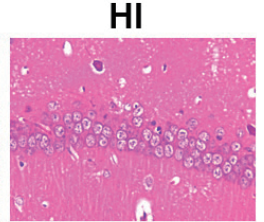

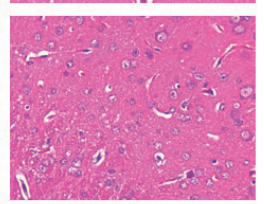

HI+CUR HI+CUR+OV-VEGF
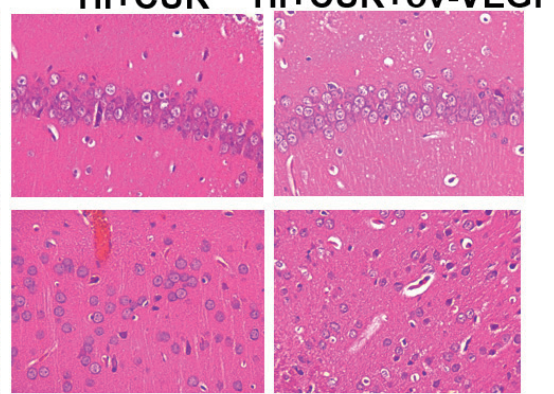
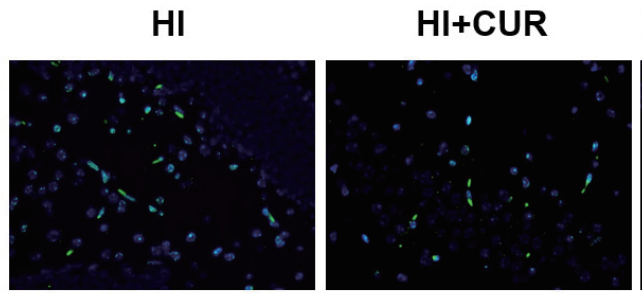

HI+CUR+ov-VEGF
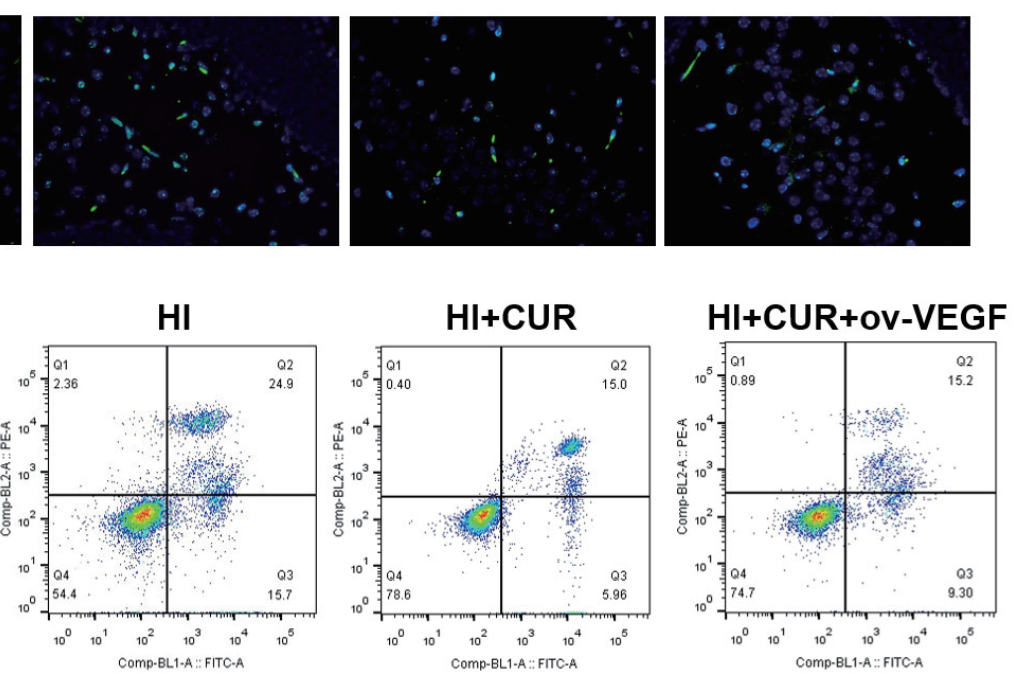

Fig. 4. The inhibitory effect of curcumin on brain $\mathrm{HI}$ damage is counteracted by VEGF overexpression in neonatal rats ( $n=12$ per group). (A) The VEGF expression in the sham, HI, HI + CUR, and HI + CUR + ov-VEGF groups by western blotting. (B) HI damage in brain tissues of the sham, HI, $\mathrm{HI}+\mathrm{CUR}$, and HI + CUR + ov-VEGF groups by TUNEL staining. (C) Neurological deficits evaluated in the sham, $\mathrm{HI}, \mathrm{HI}+\mathrm{CUR}$, and HI + CUR + ov-VEGF groups by Longa's score at $24 \mathrm{~h}$ and $72 \mathrm{~h}$ after treatment. (D) Histopathological changes of brain tissues in the sham, $\mathrm{HI}, \mathrm{HI}+\mathrm{CUR}$, and $\mathrm{HI}+\mathrm{CUR}+\mathrm{ov}-$ VEGF groups by H\&E staining ( $\times 400)$. (E) Cell apoptosis in the sham, HI, HI + CUR, and HI + CUR + ov-VEGF groups by TUNEL staining ( $\times 400)$. (F) Early and late apoptotic in brain tissues of the sham, $\mathrm{HI}, \mathrm{HI}+\mathrm{CUR}$, and $\mathrm{HI}+\mathrm{CUR}+$ ov-VEGF groups by flow cytometry. HI, hypoxic-ischemic injury; CUR, curcumin;VEGF, vascular endothelial growth factor. ${ }^{* *} \mathrm{p}<0.01$ vs. sham group; ${ }^{\# \#} \mathrm{p}<0.01$ vs. HI group.

Moreover, pretreatment with curcumin remarkably reduced the expression of p-PI3K, p-Akt, and VEGF, while IGF-1 rescued the curcumin-induced down-regulated expression of p-PI3K, p-Akt, and VEGF. In addition, VEGF injection counteracted the inhibitory effect of curcumin on brain HI damage.

Curcumin has been demonstrated to improve several neurological diseases, such as subarachnoid hemorrhage, ischemic stroke, and Alzheimer's disease, via alleviating neuroinflammation and neurological injury [22]. This study found that pretreatment with curcumin alleviated neurological deficits, hemodynamic changes, and histopathological alterations induced by brain HI damage. This suggests that curcumin was able to protect against brain HI damage in neonatal rats. Yu et al. [23] have demonstrated that curcumin attenuated hypoxia-hypercapnia brain damage- 
induced apoptosis and brain edema in rat models. Wang et al. [24] have reported that curcumin attenuates brain injuries induced by chronic intermittent hypoxia via regulating the expression of aquaporin 4 and the p38 MAPK pathway. In addition, Joseph et al. [25] have proved that nonencapsulated curcumin exerts effective neuroprotection in neonatal rats with HIE. Our study is consistent with all these previous studies that demonstrate a neuroprotective effect of curcumin on brain HI damage.

Currently, the underlying mechanisms of the action of curcumin during brain HI damage are yet to be elucidated thoroughly. Liu et al. [26] have shown that curcumin contributes to mitochondrial biogenesis, which may lead to the preventive effect of curcumin on cerebral ischemia reperfusion injury. Furthermore, blocking the TLR4/MyD88/NF-кB signaling pathway may be responsible for inhibiting experimental traumatic brain injury by curcumin via attenuating acute inflammatory injury [27]. Thus, the precise mechanisms of curcumin action in brain injury may be different in different models. This study revealed that VEGF injection counteracted the beneficial effect of curcumin on brain HI damage. After brain injury, VEGF expression has been observed in the central nervous system [28,29]. VEGF can also be induced by hypoxia that results in cerebral edema [30]. Several studies have demonstrated that exogenously administered VEGF has neuroprotective effects on brain HI damage in neonatal rats [20] and that VEGF can alleviate neurological damage by inhibiting neuronal and glial damage [31], increasing the subventricular zone size [32], and promoting neurogenesis [33]. Of note, the relationship between curcumin and VEGF has been investigated in several diseases. For example, Pan et al. [34] have reported that curcumin reduces cell growth via inhibiting VEGF expression in hepatocellular carcinoma. Lu et al. [35] have shown that curcumin can induce human pterygium fibroblast apoptosis via targeting VEGF expression. In addition, another study has also demonstrated that the anti-tumor effect of curcumin is closely associated with the significantly down-regulated VEGF in thoracic aortic aneurysms [36]. All these studies suggest an inhibitory effect of curcumin on VEGF expression, which is consistent with our study. These results indicate that pretreatment with curcumin might attenuate brain HI damage via targeting VEGF.

Furthermore, the present study found that pretreatment with curcumin inhibited the PI3K/Akt signaling pathway, while IGF1 treatment activated this pathway and VEGF expression. It has been demonstrated before that IGF-1 can activate the PI3K/ Akt signaling pathway [37], play an important role in neuronal proliferation and differentiation [38], and exert neuroprotective effects on brain HI damage in neonatal rats [39]. It has also been reported that the PI3K/Akt signaling pathway participates in angiogenesis [39], and that its activation can induce VEGF expression [40]. Our study revealed that activating the PI3K/Akt signaling pathway inhibited the neuroprotective effect of curcumin in neonatal rats with brain HI damage. Similarly, recent studies have also demonstrated that activation of the PI3K/Akt signaling pathway contributes to the recovery of neurological function after brain HI damage in neonatal rats [41] and neonatal rabbits [42]. Collectively, we postulate that pretreatment with curcumin protects against brain HI damage by targeting VEGF via the PI3K/ Akt signaling pathway.

In conclusion, the findings of this study suggest that pretreatment with curcumin improved brain $\mathrm{HI}$ injury in neonatal rats, which is likely associated with VEGF via the PI3K/Akt signaling pathway.

However, one limitation of this study is that the more information on the role of VEGF in the effect of curcumin on brain HI damage needs to be explored in the further study. Another limitation of this study is the controversy about the use of curcumin in pregnant women. Hence, further studies will aim to determine the safe dose of curcumin and its optimal administration, especially during pregnancy. In the future, we will also study whether administration of curcumin may rescue individuals in the early stages of hypoxia-induced changes.

\section{ACKNOWLEDGEMENTS}

This study was supported by the Key Research and Development Program of Hebei Province Self-funded (Project Number: 172777186), the Medical Science Research Project of Hebei Province in 2019 Project (Number: 20190933) and the Baoding Social Development Project (Number: 18ZF290).

\section{CONFLICTS OF INTEREST}

The authors declare no conflicts of interest.

\section{REFERENCES}

1. Douglas-Escobar M, Weiss MD. Hypoxic-ischemic encephalopathy: a review for the clinician. JAMA Pediatr. 2015;169:397-403.

2. Logitharajah P, Rutherford MA, Cowan FM. Hypoxic-ischemic encephalopathy in preterm infants: antecedent factors, brain imaging, and outcome. Pediatr Res. 2009;66:222-229.

3. Kharoshankaya L, Stevenson NJ, Livingstone V, Murray DM, Murphy BP, Ahearne CE, Boylan GB. Seizure burden and neurodevelopmental outcome in neonates with hypoxic-ischemic encephalopathy. Dev Med Child Neurol. 2016;58:1242-1248.

4. Lundgren C, Brudin L, Wanby AS, Blomberg M. Ante- and intrapartum risk factors for neonatal hypoxic ischemic encephalopathy. $J$ Matern Fetal Neonatal Med. 2018;31:1595-1601.

5. Northington FJ, Chavez-Valdez R, Martin LJ. Neuronal cell death in neonatal hypoxia-ischemia. Ann Neurol. 2011;69:743-758.

6. Shankaran S. Hypoxic-ischemic encephalopathy and novel strategies for neuroprotection. Clin Perinatol. 2012;39:919-929.

7. Dixon BJ, Reis C, Ho WM, Tang J, Zhang JH. Neuroprotective strategies after neonatal hypoxic ischemic encephalopathy. Int J Mol Sci. 
2015;16:22368-22401.

8. Ek CJ, D’Angelo B, Baburamani AA, Lehner C, Leverin AL, Smith PL, Nilsson H, Svedin P, Hagberg H, Mallard C. Brain barrier properties and cerebral blood flow in neonatal mice exposed to cerebral hypoxia-ischemia. JCereb Blood Flow Metab. 2015;35:818-827.

9. Storkebaum E, Lambrechts D, Carmeliet P. VEGF: once regarded as a specific angiogenic factor, now implicated in neuroprotection. Bioessays. 2004;26:943-954.

10. Silverman WF, Krum JM, Mani N, Rosenstein JM. Vascular, glial and neuronal effects of vascular endothelial growth factor in mesencephalic explant cultures. Neuroscience. 1999;90:1529-1541.

11. Guo H, Zhou H, Lu J, Qu Y, Yu D, Tong Y. Vascular endothelial growth factor: an attractive target in the treatment of hypoxic/ischemic brain injury. Neural Regen Res. 2016;11:174-179.

12. Howes MR, Fang R, Houghton PJ. Effect of Chinese herbal medicine on Alzheimer's disease. Int Rev Neurobiol. 2017;135:29-56.

13. Ip FC, Zhao YM, Chan KW, Cheng EY, Tong EP, Chandrashekar O, Fu GM, Zhao ZZ, Ip NY. Neuroprotective effect of a novel Chinese herbal decoction on cultured neurons and cerebral ischemic rats. BMC Complement Altern Med. 2016;16:437.

14. Huang L, Chen C, Zhang X, Li X, Chen Z, Yang C, Liang X, Zhu G, $\mathrm{Xu} Z$. Neuroprotective effect of curcumin against cerebral ischemiareperfusion via mediating autophagy and inflammation. J Mol Neurosci. 2018;64:129-139.

15. Cui X, Song H, Su J. Curcumin attenuates hypoxic-ischemic brain injury in neonatal rats through induction of nuclear factor erythroid-2-related factor 2 and heme oxygenase-1. Exp Ther Med. 2017;14:1512-1518.

16. Huang Y, Mao Y, Li H, Shen G, Nan G. Knockdown of Nrf2 inhibits angiogenesis by downregulating VEGF expression through PI3K/ Akt signaling pathway in cerebral microvascular endothelial cells under hypoxic conditions. Biochem Cell Biol. 2018;96:475-482.

17. Ren X, Ma H, Zuo Z. Dexmedetomidine postconditioning reduces brain injury after brain hypoxia-ischemia in neonatal rats. J Neuroimmune Pharmacol. 2016;11:238-247.

18. Jiang J, Wang W, Sun YJ, Hu M, Li F, Zhu DY. Neuroprotective effect of curcumin on focal cerebral ischemic rats by preventing blood-brain barrier damage. Eur J Pharmacol. 2007;561:54-62.

19. Rong Z, Pan R, Chang L, Lee W. Combination treatment with ethyl pyruvate and IGF-I exerts neuroprotective effects against brain injury in a rat model of neonatal hypoxic-ischemic encephalopathy. Int J Mol Med. 2015;36:195-203.

20. Feng Y, Rhodes PG, Bhatt AJ. Neuroprotective effects of vascular endothelial growth factor following hypoxic ischemic brain injury in neonatal rats. Pediatr Res. 2008;64:370-374.

21. Belinga VF, Wu GJ, Yan FL, Limbenga EA. Splenectomy following MCAO inhibits the TLR4-NF- $\mathrm{B}$ signaling pathway and protects the brain from neurodegeneration in rats. J Neuroimmunol. 2016;293:105-113.

22. Sundar Dhilip Kumar S, Houreld NN, Abrahamse H. Therapeutic potential and recent advances of curcumin in the treatment of aging-associated diseases. Molecules. 2018;23:835.

23. Yu L, Fan Y, Ye G, Li J, Feng X, Lin K, Dong M, Wang Z. Curcumin inhibits apoptosis and brain edema induced by hypoxia-hypercapnia brain damage in rat models. Am J Med Sci. 2015;349:521-525.

24. Wang B, Li W, Jin H, Nie X, Shen H, Li E, Wang W. Curcumin attenuates chronic intermittent hypoxia-induced brain injuries by in- hibiting AQP4 and p38 MAPK pathway. Respir Physiol Neurobiol. 2018;255:50-57.

25. Joseph A, Wood T, Chen CC, Corry K, Snyder JM, Juul SE, Parikh $P$, Nance E. Curcumin-loaded polymeric nanoparticles for neuroprotection in neonatal rats with hypoxic-ischemic encephalopathy. Nano Res. 2018;11:5670-5688.

26. Liu L, Zhang W, Wang L, Li Y, Tan B, Lu X, Deng Y, Zhang Y, Guo $\mathrm{X}, \mathrm{Mu}$ J, Yu G. Curcumin prevents cerebral ischemia reperfusion injury via increase of mitochondrial biogenesis. Neurochem Res. 2014;39:1322-1331.

27. Zhu HT, Bian C, Yuan JC, Chu WH, Xiang X, Chen F, Wang CS, Feng H, Lin JK. Curcumin attenuates acute inflammatory injury by inhibiting the TLR4/MyD88/NF- $\mathrm{B}$ signaling pathway in experimental traumatic brain injury. J Neuroinflammation. 2014;11:59.

28. Dore-Duffy P, Wang X, Mehedi A, Kreipke CW, Rafols JA. Differential expression of capillary VEGF isoforms following traumatic brain injury. Neurol Res. 2007;29:395-403.

29. Chaitanya GV, Cromer WE, Parker CP, Couraud PO, Romero IA, Weksler B, Mathis JM, Minagar A, Alexander JS. A recombinant inhibitory isoform of vascular endothelial growth factor164/165 aggravates ischemic brain damage in a mouse model of focal cerebral ischemia. Am J Pathol. 2013;183:1010-1024.

30. Baburamani AA, Castillo-Melendez M, Walker DW. VEGF expression and microvascular responses to severe transient hypoxia in the fetal sheep brain. Pediatr Res. 2013;73:310-316.

31. Sköld MK, Risling M, Holmin S. Inhibition of vascular endothelial growth factor receptor 2 activity in experimental brain contusions aggravates injury outcome and leads to early increased neuronal and glial degeneration. Eur J Neurosci. 2006;23:21-34.

32. Gotts JE, Chesselet MF. Vascular changes in the subventricular zone after distal cortical lesions. Exp Neurol. 2005;194:139-150.

33. Moriyama Y, Takagi N, Hashimura K, Itokawa C, Tanonaka K. Intravenous injection of neural progenitor cells facilitates angiogenesis after cerebral ischemia. Brain Behav. 2013;3:43-53.

34. Pan Z, Zhuang J, Ji C, Cai Z, Liao W, Huang Z. Curcumin inhibits hepatocellular carcinoma growth by targeting VEGF expression. Oncol Lett. 2018;15:4821-4826.

35. Lu CW, Hao JL, Yao L, Li HJ, Zhou DD. Efficacy of curcumin in inducing apoptosis and inhibiting the expression of VEGF in human pterygium fibroblasts. Int J Mol Med. 2017;39:1149-1154.

36. Li X, Fang Q, Tian X, Wang X, Ao Q, Hou W, Tong H, Fan J, Bai S. Curcumin attenuates the development of thoracic aortic aneurysm by inhibiting VEGF expression and inflammation. Mol Med Rep. 2017;16:4455-4462.

37. Cardona-Gomez GP, Mendez P, Garcia-Segura LM. Synergistic interaction of estradiol and insulin-like growth factor-I in the activation of PI3K/Akt signaling in the adult rat hypothalamus. Brain Res Mol Brain Res. 2002;107:80-88.

38. Aberg ND, Brywe KG, Isgaard J. Aspects of growth hormone and insulin-like growth factor-I related to neuroprotection, regeneration, and functional plasticity in the adult brain. ScientificWorldJournal. 2006;6:53-80.

39. Guan J, Mathai S, Liang HP, Gunn AJ. Insulin-like growth factor-1 and its derivatives: potential pharmaceutical application for treating neurological conditions. Recent Pat CNS Drug Discov. 2013;8:142160.

40. Sun L, Huang T, Xu W, Sun J, Lv Y, Wang Y. Advanced glycation 
end products promote VEGF expression and thus choroidal neovascularization via Cyr61-PI3K/AKT signaling pathway. Sci Rep. 2017;7:14925.

41. Ye L, Wang X, Cai C, Zeng S, Bai J, Guo K, Fang M, Hu J, Liu H, Zhu L, Liu F, Wang D, Hu Y, Pan S, Li X, Lin L, Lin Z. FGF21 promotes functional recovery after hypoxic-ischemic brain injury in neona- tal rats by activating the PI3K/Akt signaling pathway via FGFR1/ B-klotho. Exp Neurol. 2019;317:34-50.

42. Luo Z, Zhang M, Niu X, Wu D, Tang J. Inhibition of the PI3K/Akt signaling pathway impedes the restoration of neurological function following hypoxic-ischemic brain damage in a neonatal rabbit model. J Cell Biochem. 2019;120:10175-10185. 